\title{
Bioanalysis
}

\section{Microfluidic devices in diagnostics: what does the future hold?}

\author{
"Key to enabling early disease detection is the availability of diagnostic \\ and prognostic biomarkers, as well as of adequate technologies that enable \\ cost-effective diagnostics, discovery and large-scale population screening \\ applications."
}

Keywords: biomarkers $\bullet$ diagnostics $\bullet$ microfluidics $\bullet$ miniaturization $\bullet$ point-of-care

\section{Diagnostics}

Devastating illnesses such as cancer, immune/infectious diseases, cardiovascular and neurological disorders claim and affect the lives of millions of people each year. Early detection is key to increasing survivability rates, preventing the onset of debilitating symptoms and various risk factors, improving disease management and treatment outcomes, and, ultimately, to reducing emotional and financial burden. Key to enabling early disease detection is the availability of diagnostic and prognostic biomarkers, as well as of adequate technologies that enable cost-effective diagnostics, discovery and large-scale population screening applications. For example, in the case of cancer, detection technologies have evolved from classical immunohistochemical staining (IHS), fluorescent in situ hybridization (FISH), tissue monitoring, genetic testing (BRCA1/BRCA2 mutations) or protein assaying (PSA-prostate cancer, CA125ovarian/colon cancer), to more advanced approaches that involve searching for circulating tumor cells (CTCs), and, rather recently, toward technologies involving the use of qRT-PCR, microarrays (DNA, protein, antigen, lectin, tissue), nanoprobes, and of next generation sequencing (NGS) and microfluidic and mass spectrometry (MS) platforms. Likewise, the molecular markers have evolved from single gene/ single protein species to comprehensive panels that include whole-genome expression, transcriptional (RNA, miRNA), epigenetic (DNA methylation) or other omic profiles (proteome, secretome, exosome, glycome, metabolome). Altogether, it has become evident that information-rich data will also support the development of system-level computational models that can help elucidate not only the molecular pathways that control cell functionality, but also the identification of novel biomarkers and drug targets. As a result, the development of modern technologies that will enable the generation of such data in a discovery, screening or diagnostic context is essential to advancing our ability to detect, prevent and manage a disease.

\section{Advent of microfluidics in diagnostics}

Since its inception in the late 1980s, microfluidics has emerged as one of the most significant trends in bioanalytical instrumentation. Distinguishing features such as the ability to process very small sample amounts and volumes $(\mathrm{pL}-\mathrm{nL}$ ) in short analysis times (min or s), amenability to integration/multiplexing and high-throughput analysis, automation, small foot-print, cost, disposability and portability, have quickly bolstered the applicability of microfluidic devices in diagnostics [1-10]. Salient design configurations and novel physical principles and phenomena that become feasible only at the microscale level have further increased the appeal in the biomedical world. Devices made of silicon or glass, but mainly paper or polymeric materials have been developed for a multitude of applications. The commercial landscape has flourished accordingly, with dozens of companies seeking today the advance of microfluidics-based diagnostic technologies [1].

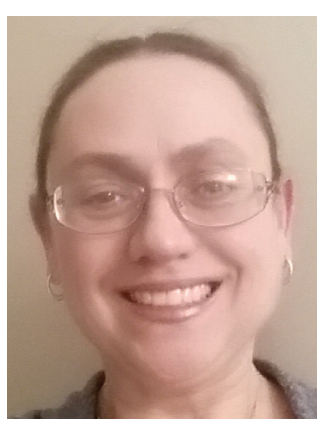

Iulia M Lazar

Department of Biological Sciences, Virginia Tech, 1981 Kraft Drive, Blacksburg, VA 24061, USA malazar@vt.edu 


\section{Present status}

Operational characteristics

An ideal device for point-of-care (POC) diagnostics is envisioned to have the ability to quantify multiple analytes, within minutes, at (sub)fmol detection levels, from $1 \mu \mathrm{l}$ bodily fluid, and transfer the results to an electronic database $[1,10]$. While such a device does not exist yet, substantial advances have been made in the development of platforms that perform chemical reactions [3], biological sample processing and analysis [2,4], cell isolation or sorting [9], and also in the development of functional elements for pumping/valving, mixing, filtering, heating, sensing and signal transducing [2,3]. Such microfluidic platforms are made primarily of polymeric materials, paper or silicon, and have been mainly developed for cancer immunodiagnostics, nucleic acid testing, digital PCR and detection of CTCs. These devices perform rather simple operations that involve the manipulation of nanodroplets, cell sorting/lysis, sample purification, filtration, extraction, centrifugation, concentration on magnetic particles and detection via colorimetric, fluorescence, chemiluminescence or electrochemical approaches, but not complex assays 'altogether and at once' [1]. Fluidic manipulations are accomplished by using capillary, electric, magnetic, gravitational, centrifugal, pressure and acoustical forces, or by droplet generation by passive (T-junction and flow focusing) or active methods (electrowetting on dielectric and dielectrophoresis) [10]

\section{Diagnostic applications}

POC or home-use self-diagnostic devices are rather simple in construction, rely mostly on capillary forces to drive $\mu \mathrm{l}$-volumes of fluids, and are fully integrated in regard to sample loading, processing and detection. Such devices have either no sophisticated readout system incorporated in the device (e.g., pregnancy immunoassay strips), or have a simple, hand-held, for example electrochemical-based detection read-out that provides a (semi)quantitative assessment for the presence of a biochemical species of interest (e.g., blood glucose monitoring). In contrast, microfluidic devices for lab diagnostics may perform complex assays, in a multiplexed format, using a variety of forces for fluidic actuation, and accommodate preanalysis robotic processing of larger volumes of sample and postanalysis sensitive and quantitatively-accurate detection. For example, the iSTAT device (presently commercialized by Abbott), Epocal's Flexcard or Abaxis' compact-disc blood analyzers use pneumatic, electroosmotic, capillary or centrifugal forces and electrochemical or photometric detection to manipulate 50-100 $\mu$ l volumes of blood and facilitate the detection and quantitation of electrolytes, gas, small molecules or protein markers of metabolic, liver, renal or cardiac disorders [1]. Furthermore, academic research has led to the development of unique and sophisticated configurations that enable complex molecular and genomic assays that involve cell lysis, DNA extraction, sequencing/sizing, PCR amplification or SNPs scoring. Such devices incorporate the full arsenal of functional and fluid actuation elements developed for bioanalytical lab-on-a-chip (LOC) or micro-total-analysis systems ( $\mu$ TAS). Major landmarks that worth highlighting include, for example, the ability to handle pL-size sample volumes [11,12], perform sample injection, separation, labeling and detection within seconds or minutes [13], or complete high-throughput DNA sequencing on multiplexed devices with as many as 768 processing lines [14]. In parallel, corporate efforts have led to the development of high-throughput microfluidic or massively parallel NGS-based molecular assays. For example, Fluidigm's microfluidic integrated arrays enable single cell gene expression analysis, genotyping or PCR. The Access Array $^{\mathrm{TM}}$, which uses a microfluidic chip that can perform 2304 unique PCRs for producing amplicon libraries for 48 unique samples in half a day, followed by NGS, was used for scanning for gene mutations that lead to familiar hypercholesterolemia [15]. While not using microfluidic technology, per se, next-gen sequencers use droplet microreactors, pL-volume wells (Roche 454) and/or advanced miniaturized sensor technologies that translate chemical signals into digital information to enable DNA sequencing and variant detection in millions of wells in just a few hours (Life Technologies' Ion Torrent ${ }^{\mathrm{TM}}$ NGS technology).

\section{Future perspective}

\section{Impacting factors}

Given the broad interest in addressing human health related issues across various societal factions, the further development of microfluidic technologies for diagnostic applications is expected to flourish and flood our everyday life. The driving questions that will guide the development of such devices will center around: (a) area of applicability (point-of-care, home-based, field, clinical lab or research-oriented bioanalysis effort), (b) type of biological material to be analyzed (blood, plasma, saliva, tear, urine, breath, food, etc.), (c) type of assay (cell, pathogen or molecular diagnostics), (d) throughput (slow discovery-type work, high-throughput population screening, sole-event diagnostics), (e) complexity and ease of fabrication (choice of material, passive vs active fluidic pumping, number of bioanalytical processing steps, detection and read-out method), (f) level of training needed for operation (advanced degree, minimally or nontrained personnel), (g) country of applicability (advanced economy or developing 
world) and (h) national and international regulatory constraints. In terms of figures of merit, the proposed devices will have to meet adequate requirements of analytical sensitivity and detection limit, throughput, speed, cross-talk free multiplexing, portability, disposability, ease of use, reliability and cost. Diagnostic sensitivity/specificity will be additional critical features for disease testing applications.

\section{Implementation}

The cost of microfabrication is decreasing as going from silicon/glass (photolithography, thin film deposition, LIGA-German acronym for lithography/electroplating and molding, wet/dry etching, bonding) to polymeric (injection molding, hot embossing, stamping, mirocontact printing, lamination, bonding), paper (photolithography/wax/inkjet-printing for hydrophobic or hydrophilic patterning, PDMS application, laser/plasma treatment, cutting) or even cotton thread (sewing, knitting or weaving which confines the fluidic flow to one dimension) devices [10,16,17]. As a result, it is expected that cheap $\mu$ PADs (paperbased analytical devices) preloaded with reagents and colorimetric visualization capabilities will be promoted first in developing countries where the need is high for preventing and containing viral, bacterial or parasitic infectious diseases (influenza, tuberculosis, Ebola, malaria, hepatitis, HIV, HPV), and for monitoring food, water, soil and air quality [16]. In countries with advanced economies, bioanalytical microfluidic devices will be developed for biomedical/biochemical and pharmaceutical research, bioterrorism prevention, high-throughput omics-based biomarker or drugtarget discovery, personalized or preventive medicine, companion diagnostics or more exotic bodily implant applications. Droplet-based or digital microfluidics, which has been already demonstrated for performing PCR [18], appears to be a good candidate for enabling single cell analysis or single molecule detection, and highly parallel, compartmentalized experiments. It is worth noting that, so far, most complex microfluidic assays have been developed for genomic applications. To improve diagnostic sensitivity and specificity, microfluidic diagnostic devices will have to incorporate complex assays for protein detection, as well, which will eventually include $1 \mathrm{D}$ or multidimensional separations with mass spectrometry detection. The advance of MS instrumentation has brought superior capabilities to deciphering the information encoded by the protein complement of a cell, and has led proteomics to unexpected heights in the past decade [19]. With already existing miniaturized mass spectrometers [20], further development of microarrayed, masstunable MS systems may enable diagnostic proteomic explorations that deliver results well beyond the present microfluidic immunoassays. Additional progress in the integration of microfluidics with advanced biosensing (SPR-surface plasmon resonance, SAWsurface acoustic waves, nanosensing), massively parallel array detection technologies or smartphone-reliant systems, wireless data transmission and cloud-based data processing/standardization/interpretation and storage, will alleviate the differences between simple diagnostic and research devices.

\section{"Point-of-care or home-use self-diagnostic devices are rather simple in construction, rely mostly on capillary forces to drive $\mu \mathrm{l}$-volumes of fluids, and are fully integrated in regard to sample loading, processing and detection."}

\section{Conclusion}

The landscape of present achievements in the field of microfluidics clearly demonstrates the potential of this technology for a broad range of healthcare and diagnostic applications. Advances in the development of various functional elements, novel operational principles and sensing, as well as continued improvements in analytical performance are ongoing. Issues related to reagent and biological sample loading/handling and storage on the chip, development of the human-chip interface, national and international regulatory barriers that prevent use, need for centralized labs for performing the analysis and costs, continue, however, to persist. Nevertheless, the multiple benefits brought by microfluidics, across the board, from research labs to POC diagnostics, encourage further investments in advancing and perfecting this technology. Simultaneously, the need for addressing the health crisis in developing countries brings a sense of urgency to fast implementation. As the technology continues to mature, as the goals continue to be refined, and as regulatory decisions become supportive of commercialization, it is expected that the effort of joint academic, government and industrial partnerships will establish microfluidics-based diagnostics into a routine practice in global healthcare.

\section{Financial \& competing interests disclosure}

This work was supported in part from NSF/DBI-1255991 grant to IM Lazar. The author has no other relevant affiliations or financial involvement with any organization or entity with a financial interest in or financial conflict with the subject matter or materials discussed in the manuscript apart from those disclosed.

No writing assistance was utilized in the production of this manuscript. 


\section{References}

1 Chin CD, Linder V, Sia SK. Commercialization of microfluidic point-of-care diagnostic devices. Lab Chip 12, 2118-2134 (2012).

2 Gervais L, De Rooij N, Delamarche E. Microfluidic chips for point-of-care immunodiagnostics. Adv. Mater. 23(24), H151-H176 (2011).

3 DeMello AJ. Control and detection of chemical reactions in microfluidics systems. Nature 442, 394-402 (2006).

4 Kim S, Kim HJ, Jeon NL. Biological applications of microfluidics gradient devices. Integr. Biol. 2, 584-603 (2010).

5 Yager P, Edwards T, Fu E et al. Microfluidics diagnostics technologies for global public health. Nature 442, 412-418 (2006).

6 Rusling JF, Kumar CV, Gutkind JS, Patel V. Measurement of biomarker proteins for point-of-care early detection and monitoring of cancer. Analyst 135(10), 2496-2511 (2010).

7 Sun J, Xianyu Y, Jiang X. Point-of-care biochemical assays using gold nanoparticle-implemented microfluidics. Chem. Soc. Rev. 43, 6239-6253 (2014).

8 Weigl B, Domingo G, LaBarre P, Gerlach J. Towards nonand minimally instrumented, microfluidics-based diagnostic devices. Lab Chip 8, 1999-2014 (2008).

9 Chen Y, Li P, Huang P-H et al. Rare cell isolation and analysis in microfluidics. Lab Chip 14, 626-645 (2014).

10 Jenkin G and Mansfield CD. Microfluidic diagnostics: methods and protocols. In: Methods in Molecular Biology. Human Press/Springer, NY, USA (2013).

11 Harrison DJ, Manz A, Fan ZH, Ludi H, Widmer HM. Capillary electrophoresis and sample injection systems integrated on a planar glass chip. Anal. Chem. 64, 1926-1932 (1992).
12 Jacobson SC, Hergenroder R, Koutny LB, Warmack RJ, Ramsey JM. Effects of injection schemes and column geometry on the performance of microchip electrophoresis devices. Anal. Chem. 66, 1107-1113 (1994).

13 Jacobson SC, Culbertson CT, Daler JE, Ramsey JM. Microchip structures for submillisecond electrophoresis. Anal. Chem. 70, 3476-3480 (1998).

14 Aborn JH, El-Difrawy SA, Novotny M et al. A 768lane microfabricated system for high-throughput DNA sequencing. Lab Chip 5, 669-674 (2005).

15 Hollants S, Redeker EJW, Matthijs G. Microfluidic amplification as a tool for massive parallel sequencing of the familial hypercholesterolemia genes. Clin. Chem. 58(4), 717-724 (2012).

16 Yetisen AK, Akram MS, Lowe CR. Paper-based microfluidic point-of-care diagnostic devices. Lab Chip 13, 2210-2251 (2013).

17 Li X, Tian J, Shen W. Thread as a versatile material for low-cost microfluidic diagnostics. ACS Appl. Mater. Interfaces 2(1), 1-6 (2010).

18 Zhang H, Jenkins G, Zou Y, Zhu Z, Chaoyong James Yang CJ. Massively parallel single-molecule and single-cell emulsion reverse transcription polymerase chain reaction using agarose droplet microfluidics. Anal. Chem. 84, 3599-3606 (2012).

19 Lesur A, Domon B. Advances in high-resolution accurate mass spectrometry application to targeted proteomics. Proteomics 15(5-6), 880-890 (2015).

20 Li L, Chen T-C, Ren Y, Hendricks PI, Cooks RG, Ouyang Z. Mini 12, miniature mass spectrometer for clinical and other applications-introduction and characterization. Anal. Chem. 86(6), 2909-2916 (2014). 